\title{
A NOTE ON LACUNARY FOURIER SERIES
}

\section{TOMIĆ}

A theorem of Kolmogoroff [2, p. 73] states that if the Fourier series $\mathfrak{S}[f]$ of an $L$-integrable function $f(x)$ has an infinity of gaps $\left(n_{\nu}, n_{\nu}^{\prime}\right)$ for which $n_{\nu}^{\prime} / n_{\nu} \geqq \lambda>1$, (gaps of Hadamard's type), then $s_{n_{\nu}} \rightarrow f$ for almost all $x$, and it can be concluded that if $f(x)$ is continuous then this is valid for all $x$. From this theorem one can derive another theorem of Kolmogoroff: If $f \in L^{2}$ and $n_{\nu+1} / n_{\nu} \geqq \lambda>1$ then $s_{n_{\nu}} \rightarrow f$ for almost all $x$. Recently R. Gosselin [1] has proved a similar theorem with considerably larger subsequences $\left(n_{\nu}, n_{\nu}^{\prime}\right)$, although with less precision in locating the indices.

In this note, we prove the following theorem for gaps where $n_{\nu}^{\prime} / n_{\nu} \rightarrow 1$.

Theorem. Let $f(x)$ be continuous at $x=x_{0}$, and let $\subseteq[f]$ be a lacunary Fourier series with an infinity of gaps $\left(n_{\nu}, n_{\nu}^{\prime}\right)$ for which $n_{\nu}^{\prime}-n_{\nu} \rightarrow \infty$, $n_{\nu}^{\prime} / n_{\nu} \rightarrow 1$ and

$$
\omega\left(x_{0}, \frac{\pi}{n_{\nu}^{\prime}-n_{\nu}}\right) \log \left(1-\frac{n_{\nu}}{n_{\nu}^{\prime}}\right) \rightarrow 0
$$

with

$$
\omega\left(x_{0}, \delta\right)=\sup _{0<|t| \leqq \delta}\left\{\left|f\left(x_{0}+t\right)-f\left(x_{0}\right)\right|\right\}
$$

then

$$
s_{n_{\nu}}\left(x_{0}\right) \rightarrow f\left(x_{0}\right) .
$$

It is well known $\left[2\right.$, p. 45] that $\left|f\left(x_{0}+h\right)-f\left(x_{0}\right)\right|=o(\log 1 /|h|)^{-1}$ does not ensure the convergence of $\mathfrak{S}[f]$ at the point $x_{0}$. From the above theorem it follows that in this case $\subseteq[f]$ converges if, for example, it is a lacunary Fourier series with $n_{\nu}=\nu^{1+\epsilon}, n_{\nu+1}=(\nu+1)^{1+\epsilon}$ for every $\epsilon>0$.

Proof of Theorem. Take $n_{\nu}=n, n_{\nu}^{\prime}=m$ and denote by $D_{n}(t)$ and $K_{n}(t)$ the Dirichlet and Fejér kernels, i.e.

$D_{n}(t)=\frac{1}{2}+\sum_{k=1}^{n} \cos k t, \quad(n+1) K_{n}(t)=\sum_{\nu=0}^{n} D_{\nu}(t)=\frac{\sin ^{2}(n+1) t / 2}{2 \sin ^{2} t / 2}$.

From the identity

Received by the editors June 10, 1959 and, in revised form, July 2, 1959. 


$$
\begin{aligned}
m K_{m-1}(t)-n K_{n-1}(t) & =\sum_{\nu=n}^{m-1} D_{\nu}(t) \\
& =(m-n) D_{n}(t)+\sum_{\nu=1}^{m-n-1}(m-n-\nu) \cos (n+\nu) t,
\end{aligned}
$$

we have in virtue of the lacunarity of $\subseteq[f]$

$$
\begin{aligned}
s_{n}-f\left(x_{0}\right) & =\frac{1}{\pi} \int_{0}^{\pi} \phi\left(x_{0}, t\right) D_{n}(t) \\
& =\frac{1}{\pi(m-n)} \int_{0}^{\pi} \phi\left(x_{0}, t\right)\left[m K_{m-1}(t)-n K_{n-1}(t)\right] d t,
\end{aligned}
$$

with

$$
\phi\left(x_{0}, t\right)=f\left(x_{0}+t\right)+f\left(x_{0}-t\right)-2 f\left(x_{0}\right) .
$$

The last integral can be written in the form

$$
\begin{gathered}
\frac{1}{2 \pi(m-n)}\left\{\int_{0}^{\pi /(m-n)}+\int_{\pi /(m-n)}^{\delta}+\int_{\delta}^{\pi}\right\} \phi\left(x_{0}, t\right) \frac{\sin ^{2} m \frac{t}{2}-\sin ^{2} n \frac{t}{2}}{\sin ^{2} \frac{t}{2}} d t \\
=I_{1}+I_{2}+I_{3}
\end{gathered}
$$

say. We have for $I_{1}$

(2) $\left|I_{1}\right| \leqq \frac{2}{\pi(m-n)} \omega\left(x_{0}, \frac{\pi}{m-n}\right) \int_{0}^{\pi / 2(m-n)} \frac{\left|\sin ^{2} m t-\sin ^{2} n t\right|}{\sin ^{2} t} d t$.

It remains to estimate

$$
I_{1}^{\prime}=\int_{0}^{\pi / 2(m-n)} \frac{\left|\sin ^{2} m t-\sin ^{2} n t\right|}{\sin ^{2} t} d t .
$$

Taking $m t=\tau, n / m=\xi$ we obtain

$$
I_{1}^{\prime} \leqq \frac{\pi^{2}}{4} m \int_{0}^{m \pi / 2(m-n)} \frac{\left|\sin ^{2} \tau-\sin ^{2} \xi \tau\right|}{\tau^{2}} d \tau .
$$

It follows from

$$
\sin ^{2} \tau-\sin ^{2} \xi \tau=\sin (1-\xi) \tau \sin (1+\xi) \tau
$$

and from 


$$
\frac{\sin \alpha t}{t} \leqq \frac{2 \alpha}{1+\alpha t},
$$

that the integral of the right-hand side of (2) is less than

$$
\begin{aligned}
\int_{0}^{\infty} \mid \sin (1-\xi) \tau \sin (1 & +\xi) \tau \mid \frac{d \tau}{\tau^{2}} \\
& \leqq 4\left(1-\xi^{2}\right) \int_{0}^{\infty} \frac{d t}{(1+(1+\xi) t)(1+(1-\xi) t)} \\
& =2\left(1-\xi^{2}\right) \frac{1}{\xi} \log \frac{1+\xi}{1-\xi} .
\end{aligned}
$$

In virtue of (2), (3) and $\xi=n / m$ we have

$$
\left|I_{1}\right| \leqq \pi \frac{m+n}{n} \omega\left(x_{0}, \frac{\pi}{m-n}\right) \log \frac{m+n}{m-n}
$$

and the last expression for $m / n \rightarrow 1$ is equivalent to the left-hand side of (1).

On the other hand, we obtain

$$
\left|I_{2}\right| \leqq \frac{2}{2 \pi(m-n)} \pi^{2} \omega\left(x_{0}, \delta\right) \frac{m-n}{\pi}=\omega\left(x_{0}, \delta\right),
$$

and

$$
\left|I_{3}\right| \leqq \frac{C}{(m-n) \delta^{2}}
$$

with an absolute constant $C$.

Given an $\epsilon$ we can choose $\delta$ so that $\omega\left(x_{0}, \delta\right)<\epsilon$ and we can suppose $(m-n)$ so large that $I_{1}+I_{2}+I_{3}=o(1)$; this proves the theorem.

\section{REFERENCES}

1. R. P. Gosselin, On the convergence of Fourier series of functions in an $L^{p}$ class, Proc. Amer. Math. Soc. vol. 7 (1956) pp. 392-397.

2. G. H. Hardy and W. W. Rogosinski, Fourier series, Cambridge, 1950.

Institut Mathématique, Belgrade, Yugoslavia 